\title{
Sur deux nouveaux Trichostrongyles, parasites du Porc-Epic, au Viet-Nam
}

\author{
Par Marie-Claude DURETTE-DESSET
}

Le matériel étudié provient de deux Hystrix cristata L. morts au Jardin Zoologique de Saïgon. Il nous a été aimablement envoyé par le $\mathrm{D}^{\mathrm{r}}$ Le Van Hoa et nous l'en remercions bien vivement.

Les deux espèces associées dans ce matériel paraissent intéressantes à décrire, d'autant plus qu'il n'y a, à notre connaissance, aucun Heligmosome connu jusqu'à maintenant chez ce Rongeur.

\section{LONGISTRIATA LEVANHOAI n. sp.}

\section{Matériel étudié :}

$-3 \sigma, 1 \%$, parasites de l'intestin d'un Hystrix cristata L. mort au Jardin Zoologique de Saïgon. Date de récolte: 16/8/1965. (Muséum Paris, Zool. Vers, tube $313 \mathrm{~K}$ ) ;

- 3 \%, parasites de l'intestin d'un Hystrix cristata L. mort au Jardin Zoologique de Saïgon. Date de récolte: 13/8/1965. Co-parasites de Longistriata cordicauda (Muséum Paris, Zool. Vers, tube $287 \mathrm{~K})$.

Description : Nématodes de petite taille, ne présentant pas d'enroulement sénestre le long de la ligne ventrale. Le ver est simplement enroulé en un tour de spire très lâche, le long de la ligne latérale droite.

Extrémité antérieure enflée en une vésicule céphalique haute de $75 \mu$.

ETUDE DES ARÊTES: La cuticule est ornée de 13 arêtes longitudinales réparties comme l'indiquent les figs 4 et 5 . Ces arêtes débutent à environ $300 \mu$ en arrière de la capsule céphalique (fig. $5, \mathrm{~A}$ ). Chez le $\delta$, les arêtes ventrales disparaissent les premières à $550 \mu$ de la bourse caudale (fig. $4, \mathrm{C}$ ), puis les arêtes dorsales et les 2 arêtes gauches à $400 \mu$ (fig. 4, D). Chez la $\uparrow$, elles disparaissent entre $500 \mu$ et $280 \mu$ de la vulve (fig. 1 , F, G). Au niveau de celle-ci, il n'y a plus aucune arête (fig. 5, H).

En coupe transversale, on s'aperçoit qu'il n'existe pas de gradient de taille des arêtes. Elles sont toutes sensiblement égales, excepté les 2 arêtes gauches très développées. La pointe des épines est dirigée vers la gauche (fig. 4, B ; 5, B). 

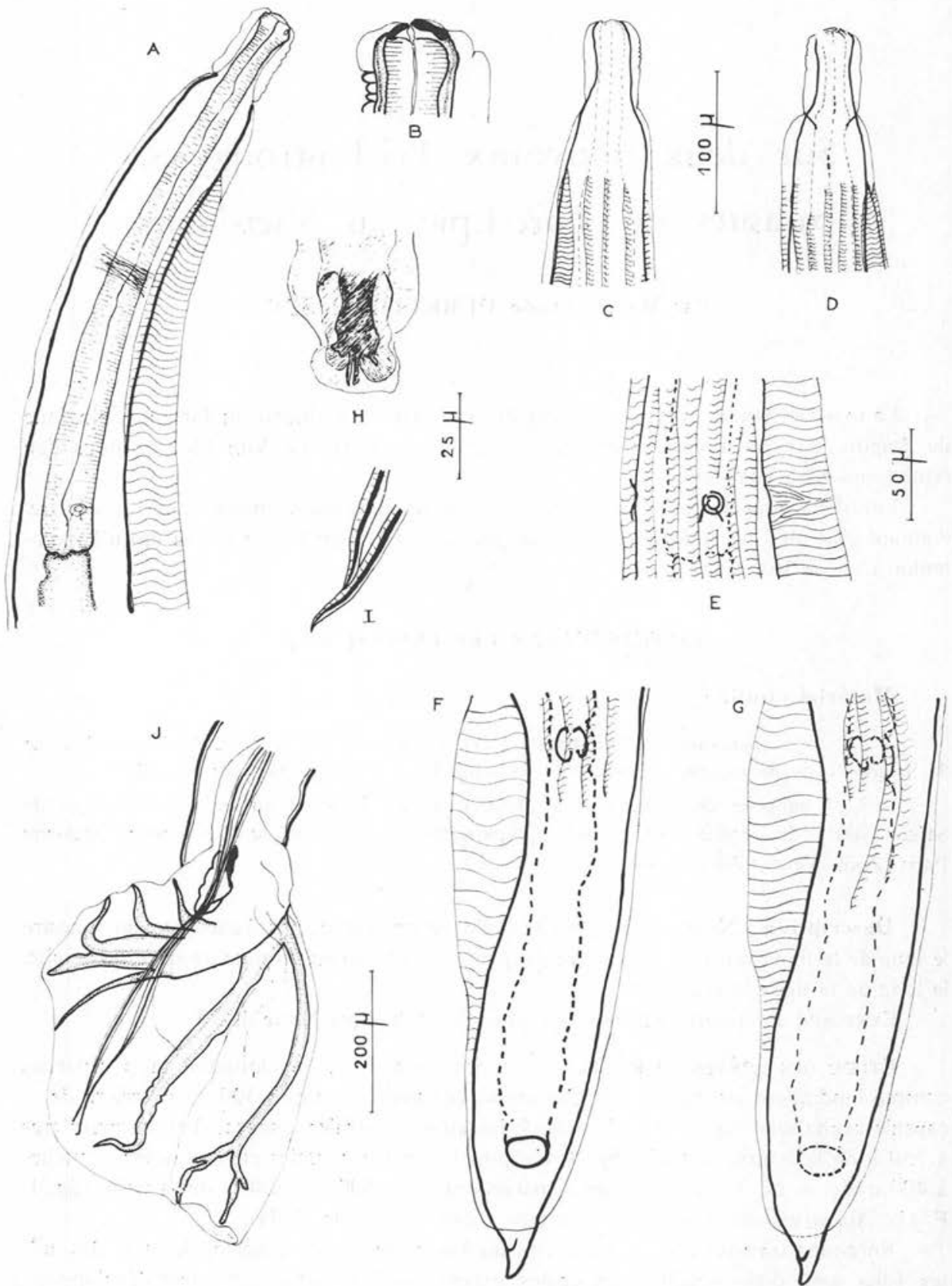

Fig. 1. - Longistriata levanhoai n. sp. A : ₹, extrémité antérieure, vue ventrale. B : $\delta$, extrémité céphalique, vue ventrale. $\mathrm{C}: \mathcal{\wp}$, extrémité antérieure, vue dorsale, montrant le départ des arêtes cuticulaires. D : ơ, extrémité antérieure, vue ventrale, montrant le départ des arêtes cuticulaires. B : $q$, vue ventrale, détail du pore excréteur et des diérides. F : $\$$, extrémité postérieure, vue dorsale en profondeur, montrant la disparition des arêtes cuticulaires du côté ventral. G : 'o, extrémité postérieure, vue dorsale montrant la disparition des arêtes cuticulaires. $\mathrm{H}$ : đó détail du cône génital. I : $\delta^{\prime}$, détail de l'extrémité des spicules. J : $\delta$, extrémité postérieure, vue latérale gauche. A, C, D : éch. $100 \mu . \mathrm{B}, \mathrm{H}, \mathrm{I}$ : éch. $25 \mu$. E : éch. $50 \mu$. F, G, J : éch. $200 \mu$ 


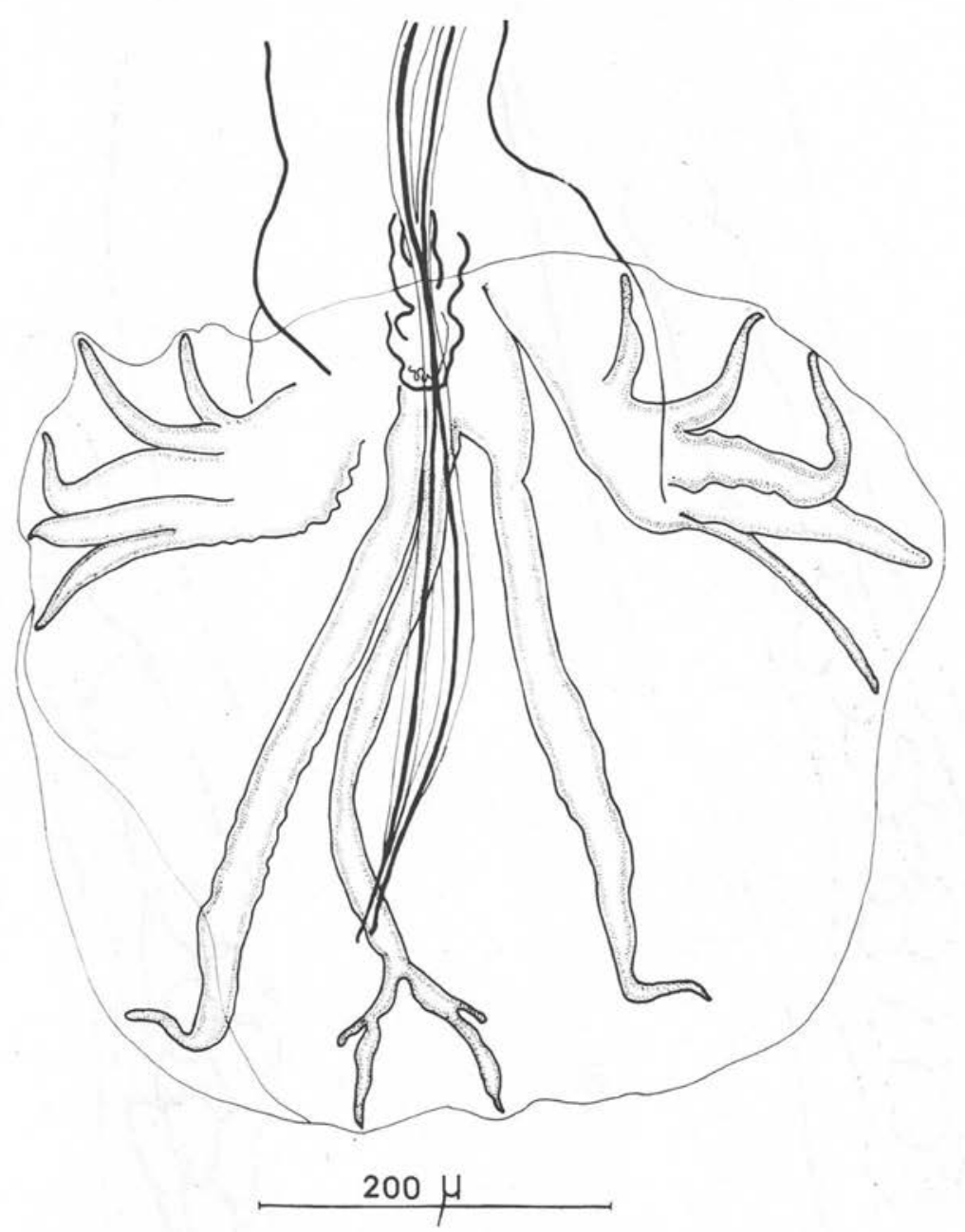

Fig. 2. - Longistriata levanhoai n. sp. $\widehat{\sigma}$ : extrémité postérieure, vue ventrale

L' « aile » gauche est très originale : ce n'est pas la formation plate, habituelle, mais une énorme vésicule, tantôt comprise entre les deux arêtes gauches hypertrophiées ( $\sigma^{7}$ : fig. $4, \mathrm{~B} ;$; : fig. $\left.5, \mathrm{C}, \mathrm{F}\right)$; tantôt débordant largement sur tout le pourtour du corps et formant un vaste cylindre à l'intérieur duquel le corps proprement dit est en position plus ou moins excentrique (fig. 5, B, D, E, G).

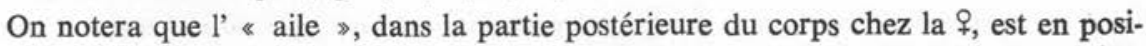
tion ventrale et non en position dorsale comme chez la plupart des espèces (fig. 3 , B). L' « aile » ne peut se rétracter, car sa cavité est emplie d'une substance particulière 

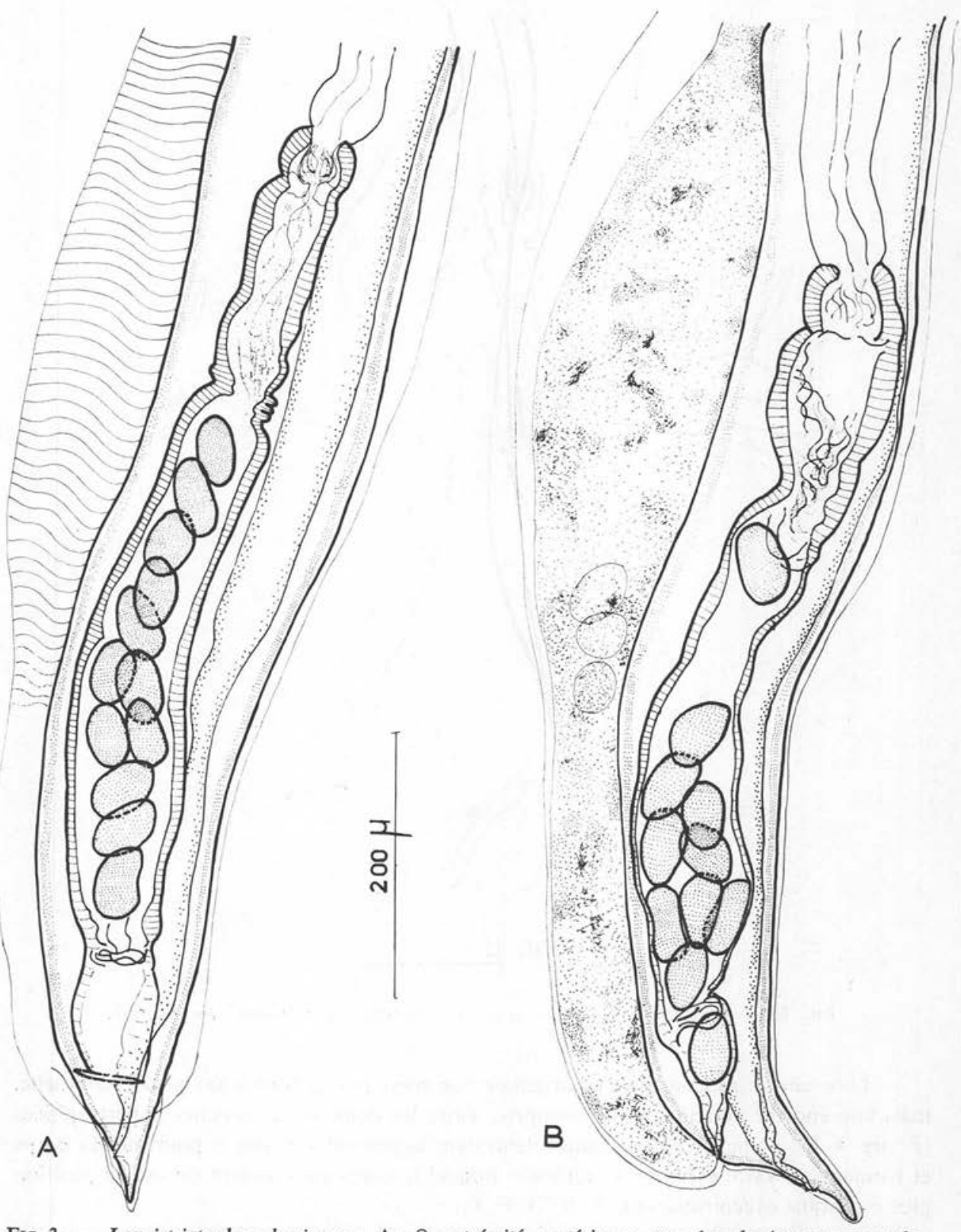

FIG 3. - Longistriata levanhoai n. sp. A: $q$, extrémité postérieure, vue dorsale (en vue ventrale, l'observation est rendue difficile par la présence des substances contenues dans la vésicule). B : autre spécimen $\mathcal{q}$, extrémité postérieure, vue latérale gauche, montrant l'importante dilatation ventrale cuticulaire 
d'aspect réticulé ou granuleux dont l'étude histologique a été effectuée pour Longistriata cordicauda (cf. p. 461).

Mâle: Corps long de $6,3 \mathrm{~mm}$, large de $180 \mu$, aile gauche comprise. Esophage long de $410 \mu$. Anneau nerveux, pore excréteur et diérides respectivement à $150 \mu$ et $130 \mu$ de l'apex.

Spicules subégaux, ailés, à extrémité pointue (fig. 1, I), longs de $1.300 \mu$. Le gubernaculum est long de $50 \mu$ sur $30 \mu$ de large ; le cône génital est figuré en $1, \mathrm{H}$.

A
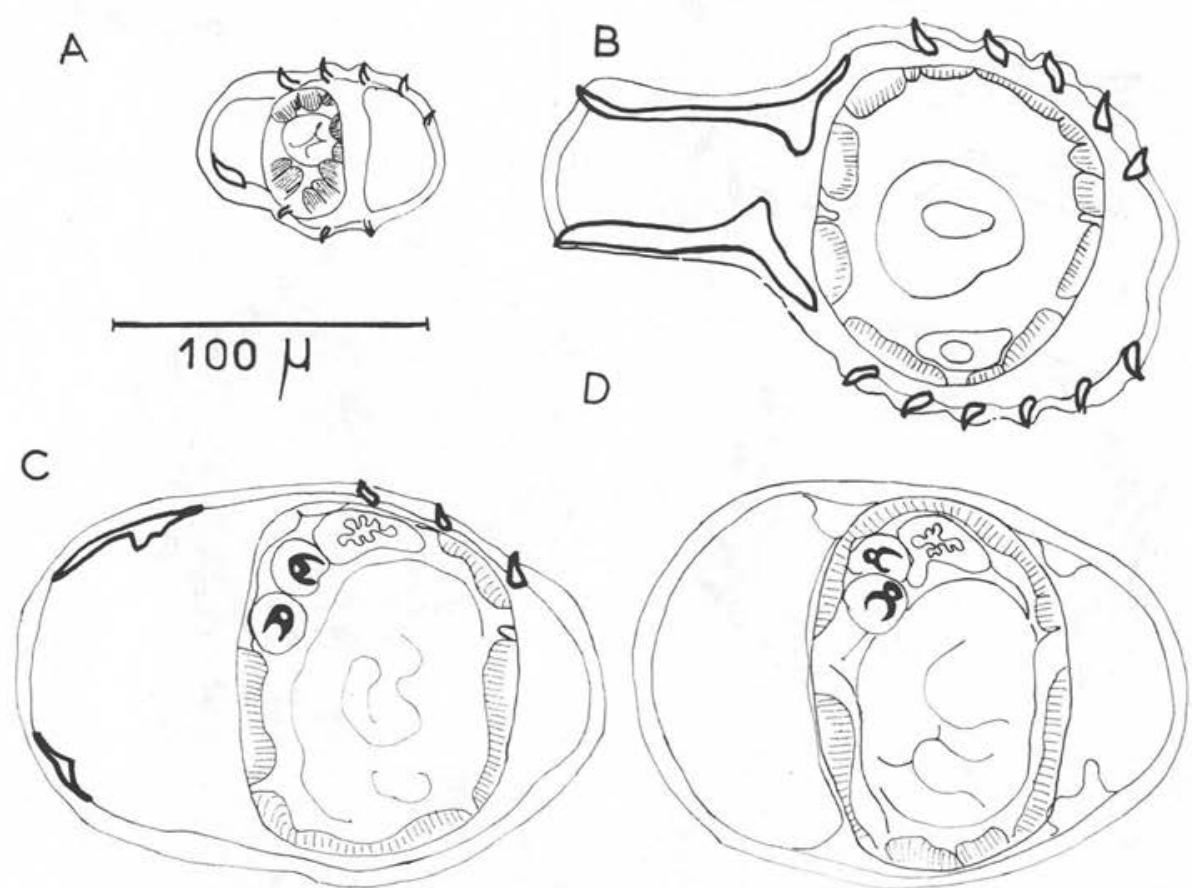

FIG. 4. - Longistriata levanhoai $\mathrm{n}$. sp. $\delta$ : coupes transversales du corps. A : à $200 \mu$ en arrière de la tête. B : au milieu du corps $(4 \mathrm{~mm})$. C : à $530 \mu$ en avant de la bourse caudale. D : à $400 \mu$ en avant de la bourse caudale

La bourse caudale est presque aussi haute que large $(495 \mu)$ (fig. 2) ; elle présente une légère asymétrie : la partie distale du lobe droit est plus développée que celle du lobe gauche, tandis que la partie proximale du lobe droit est plus petite que celle du lobe gauche. La dorsa'e est aussi légèrement asymétrique, elle est parallèle à l'externodorsale droite sur la moitié de sa longueur, puis s'infléchit vers la gauche. L'externodorsale droite est plus longue que la gauche, mais prend naissance plus bas sur la dorsale. Les autres côtes sont groupées dans la partie proximale de la bourse. Nous n'avons pas observé de papilles prébursales (fig. 2). 
A
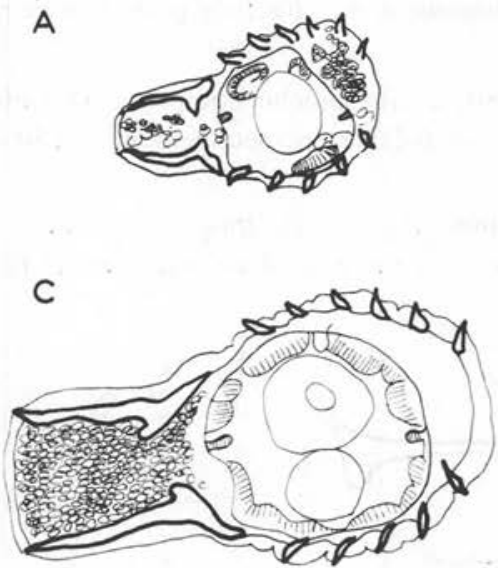

$E$

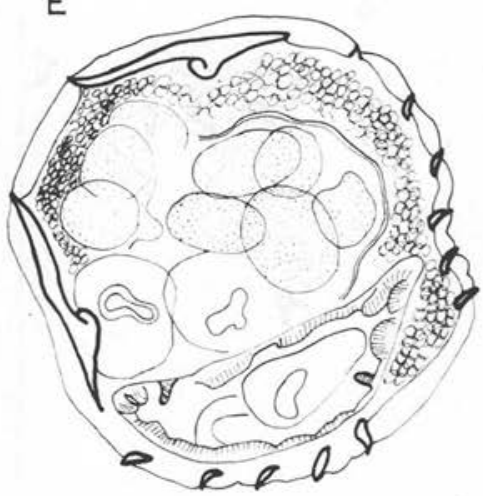

G

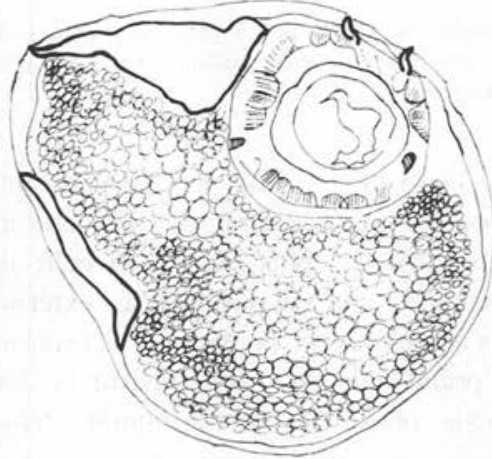

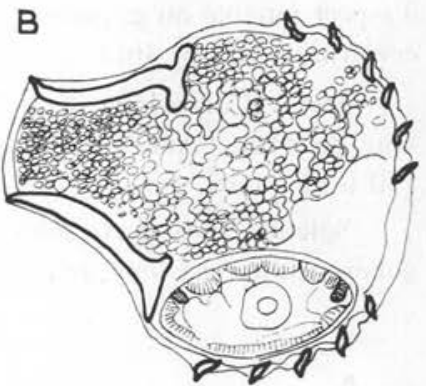

$100 \mathrm{H} \quad \mathrm{D}$
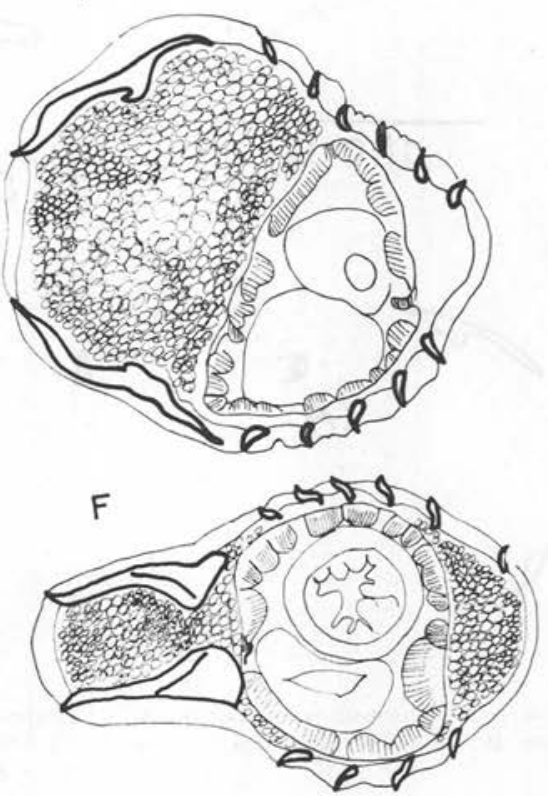

$H$

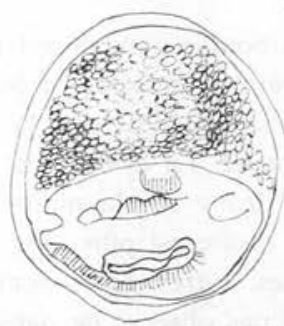

Fig. 5. - Longistriata levanhoai n. sp. $q$ : coupes transversales du corps. A : à $350 \mu$ en arrière de la tête. B : à $2.250 \mu$ en arrière de la tête. C: au milieu du corps $(4.950 \mu)$. D : à $2.380 \mu$ en avant de l'extrémité caudale. E : à $1.960 \mu$ en avant de l'extrémité caudale. F : à $660 u$ en avant de l'extrémité caudale. G : à $560 \mu$ en avant de l'extrémité caudale. $\mathrm{H}$ : à $50 \mu$ en avant de la vulve 
Femelle: Corps long de $9 \mathrm{~mm}$, large de $200 \mu$, aile gauche comprise. Esophage long de $500 \mu$. Anneau nerveux, pore excréteur et diérides situés respectivement à $310 \mu$ et $480 \mu$ de l'apex (fig. 1, A).

Appareil génital monodelphe. Vulve située à $110 \mu$ de l'extrémité postérieure, ( $210 \mu$ chez un autre spécimen), et à $65 \mu$ de l'anus. Vestibule très long $(660 \mu)$, sphincter et trompe longs respectivement de $50 \mu$ et $195 \mu$. L'utérus mesure $950 \mu$. Queue très courte $(45 \mu)$, à extrémité pointue.

Les œufs, à coque mince, mesurent environ $50 \mu \times 35 \mu$ (fig. 3, A, B).

Discussion : Nos parasites présentent les caractères essentiels du genre Longistriata sensu Chabaud 1959.

Quoique le système des arêtes cuticulaires paraisse original, il est insuffisamment connu chez les autres espèces pour que nous puissions l'utiliser comme critère de diagnose.

Mais d'autres éléments morphologiques distinguent aisément notre matériel des autres espèces : chez la $q$, le vestibule est très long (environ $1 / 10^{\circ}$ du corps); chez le $\delta^{*}$, la bourse caudale est considérablement allongée dans le sens de la hauteur; les côtes bursales latéro-ventrales sont toutes groupées dans la partie proximale de la bourse ; la dorsale et les externo-dorsales sont très longues. D'autres genres, comme Impalaia et Viannella possèdent une dorsale et des externo-dorsales longues, mais dans ce cas, les côtes latérales sont également allongées. L'espèce type du genre Longistriata, L depressa (Dujardin, 1845) (= L. codrus Thomas, 1953) possède aussi une dorsale longue, mais les externo-dorsales sont petites.

Enfin le Ver est enroulé de façon lâche le long de la ligne latérale droite et non de la ligne ventrale comme c'est le cas général.

Ces caractères nous paraissent suffisants pour séparer notre espèce et nous proposons de la nommer Longistriata levanhoai $\mathrm{n}$. sp. en la dédiant au $\mathrm{D}^{\mathrm{r}}$ Le Van Hoa.

\section{LONGISTRIATA CORDICAUDA n. sp.}

\section{Matériel étudié :}

$3 \delta^{7}, 2$ ㅇ, parasites de l'intestin d'un Hystrix cristata L. mort au Jardin Zoologique de Saïgon le 13-8-1965. (Muséum Paris, Zool. Vers, tube 287 K). Nos spécimens sont co-parasites de Longistriata levanhoai.

Description : Nématodes de petite taille, enroulés de façon sénestre le long de la ligne ventrale. Les tours de spire sont au nombre de quatre à cinq chez le $\delta$, de cinq à six chez la $q$.

La cuticule s'élargit à son extrémité antérieure pour former une vésicule céphalique, haute de $80 \mu$ en moyenne.

Esophage assez court (environ $1 / 30^{\circ}$ du corps), dilaté postérieurement. Anneau nerveux difficile à voir, en arrière de la moitié de l'œsophage. Pore excréteur bien marqué au même niveau que les diérides, situé un peu avant la fin de l'œsophage. 
ETUDE DES ARÊTES: Les arêtes cuticulaires, longitudinales, sont au nombre de 8 . Elles débutent, presque toutes au même niveau, derrière la vésicule céphalique (fig. $9, \mathrm{~A}$ ) et s'étendent jusqu'à environ $300 \mu$ de la bourse caudale chez le $\sigma^{*}$ (fig. 9, E) et $400 \mu$ de la queue chez la $\uparrow$. En coupe transversale, la répartition de ces arêtes, dans la partie moyenne du corps, est représentée sur la fig. 9, B.

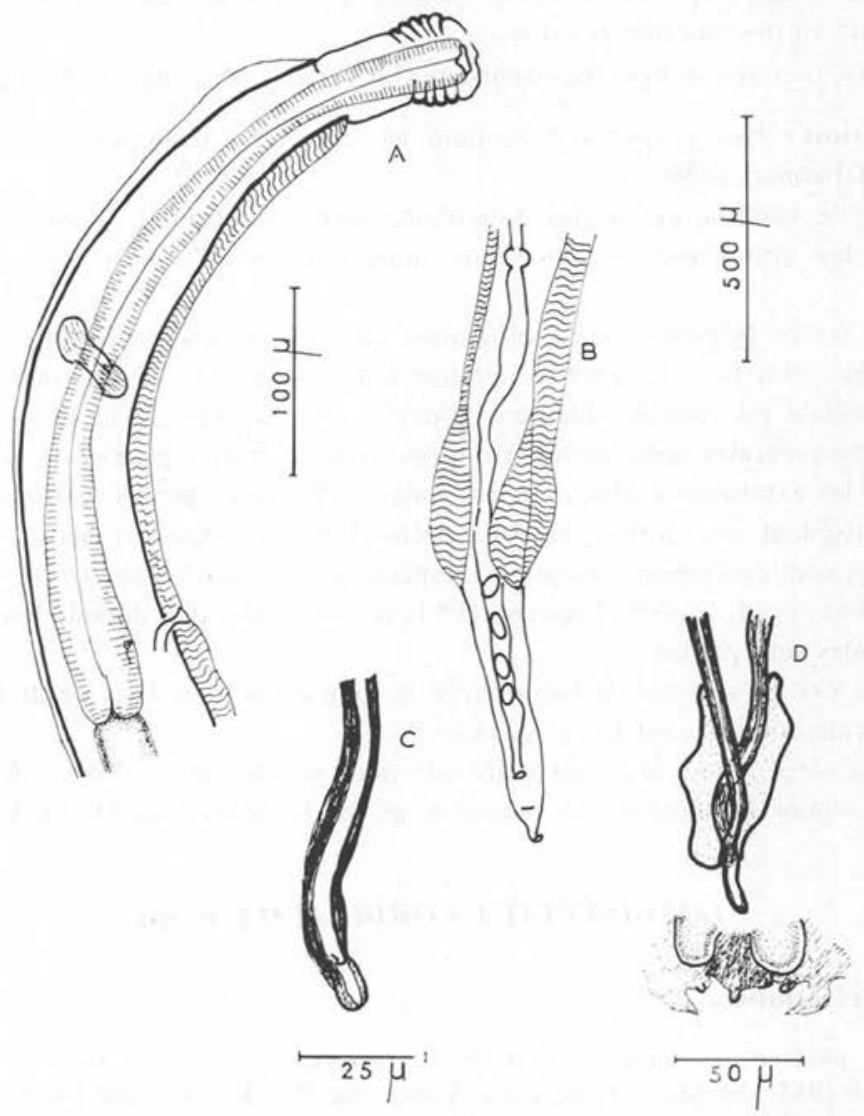

FIG. 6. - Longistriata cordicauda n. sp. A: $\delta^{\star}$, extrémité antérieure, vue latérale droite. B: $q$, extrémité postérieure, vue ventrale. C: $\hat{\delta}$, détail de l'extrémité d'un spicule. D: détail du gubernaculum et du cône génital

On notera d'une part, la présence d'une seule arête ventrale, d'autre part le grand développement des deux arêtes gauches. On remarque aussi qu'il n'existe pas de gradient de taille des épines. Elles sont toutes à peu près égales, excepté les deux gauches et la ventrale. La pointe des épines est dirigée vers la gauche (fig. 9, B).

Chez la $q$ seulement, l'arête ventrale est en réalité formée de deux arêtes accolées 
(fig. 9, A, B,C); à environ $900 \mu$ de la vulve, l'arête ventrale et l'arête dorsale proche du champ latéral droit prennent de l'importance (fig. 9, B). Elles atteignent $65 \mu$ de large à $650 \mu$ de la vulve (fig. 9, C), puis disparaissent $300 \mu$ plus loin. Au niveau de la vulve, il n'y a plus aucune arête (fig. 9 , E).

Ici, comme dans l'espèce précédente, l'espace situé entre les deux arêtes gauches (dans la région antérieure), ou bien l'espace compris entre le corps proprement dit et la cuticule dilatée (dans la région postérieure), est empli de formations granuleuses ou vésiculeuses, qui empêchent la cuticule de se replier sur elle-même. Sur coupe histologique, ces formations se colorent de la même façon que les arêtes (cf. tableau) et se présentent comme une bourre inorganisée de substances chitinoïdes.

Tableau des colorations utilisées, mettant en Évidence L'ANALOgie de Composition DES ARÊTES ET DE LA VÉSICULE

\begin{tabular}{|c|c|c|c|c|}
\hline COLORANTS & arêtes & vésicule & $\begin{array}{l}\text { cuticule } \\
\text { externe }\end{array}$ & $\begin{array}{l}\text { cuticule } \\
\text { interne }\end{array}$ \\
\hline H. Pic. Ind. Carm. . . . . & $\mathbf{P}+$ & $\mathrm{P}+$ & $\mathrm{IC}+$ & $\mathrm{IC}+$ \\
\hline $\begin{array}{l}\text { Digestion salivaire } \\
+ \text { P.A.S. } \ldots \ldots \ldots \ldots \ldots \ldots\end{array}$ & + & + & - & 一 \\
\hline Eau distillée + P.A.S. ... & + & + & + & + \\
\hline $\begin{array}{l}\text { P.A.S. avec blocage des } \\
\text { fonctions } \mathrm{CHOH}\end{array}$ & + & + & 一 & 一 \\
\hline
\end{tabular}

Mâle: Corps long de $10,1 \mathrm{~mm}$, large de $210 \mu$, aile gauche comprise. Esophage long de $365 \mu$. Anneau nerveux, pore excréteur et diérides respectivement à $265 \mu, 320 \mu$ et $325 \mu$ de l'apex (fig. 6, A).

Spicules sub-égaux, ailés, à extrémité ronde, (fig. 6, C) longs de $1.800 \mu$, glissant dans un gubernaculum long de $80 \mu$ sur $50 \mu$ de large. Le cône génital est figuré en $6, \mathrm{D}$.

La bourse caudale, étalée, a grossièrement la forme d'un cœur. Elle est plus haute $(490 \mu)$ que large (largeur maximale $430 \mu$ ), asymétrique, le lobe gauche étant plus grand que le lobe droit. De petites côtes pré-bursales sont visibles.

La dorsale est très longue. Elle donne naissance aux $2 / 3$ de sa longueur à un rameau gauche, puis à un rameau droit $125 \mu$ plus loin. Enfin, elle se divise en deux branches égales à $360 \mu$ de sa naissance. Les externo-dorsales sont épaisses. La droite prend naissance sur la dorsale, à un niveau plus proximal que la gauche. Les autres côtes sont groupées de façon assez caractéristique comme l'indique la fig. 7 .

Femelle : Corps long de $12,6 \mathrm{~mm}$, large de $220 \mu$, aile gauche comprise. Esophage long de $340 \mu$. Anneau nerveux, pore excréteur et diérides situés respectivement à $180 \mu, 270 \mu$ et $270 \mu$ de l'apex. 
Appareil génital monodelphe. Vulve située à $120 \mu$ de l'extrémité caudale et à $80 \mu$ de l'anus. L'ovéjecteur comprend un vestibule extrêmement long $(1.245 \mu)$, un sphincter de $55 \mu$ et une trompe de $220 \mu$.

Queue très courte de $40 \mu$ arrondie à son extrémité.

Les œufs mesurent $50 \times 30 \mu$. Ils ont une coque mince et se trouvent au stade morula (fig. 8).

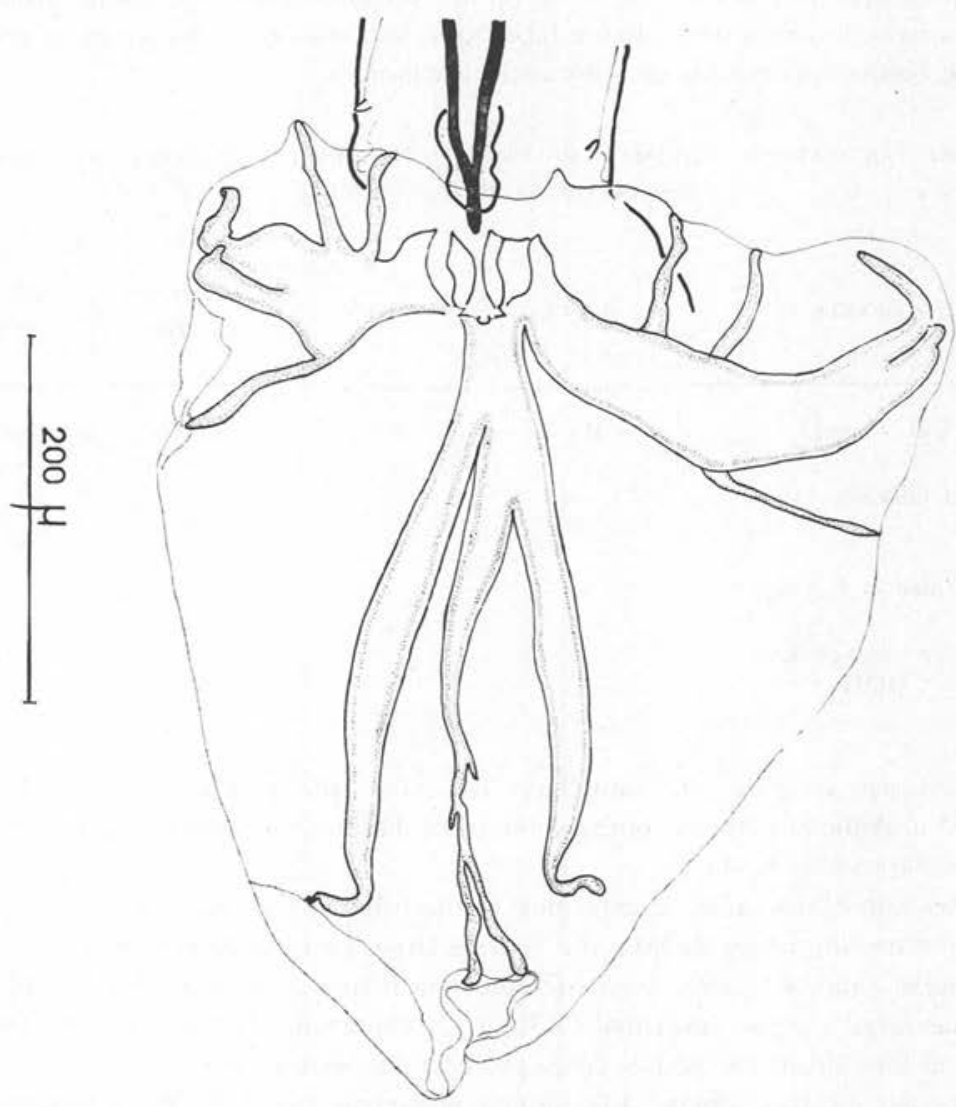

FIo. 7. - Longistriata cordicauda. $\sigma^{7}$ : extrémité postérieure, vue ventrale

Discussion : Nos parasites ont de nombreux points communs avec l'espèce précédente Longistriata levanhoai, en particulier la présence d'un vestibule très long chez la $\subsetneq$; la forme de la bourse caudale chez le $\delta^{t}$ avec toutes les côtes groupées dans la partie proximale, excepté la dorsale et les externo-dorsales très longues; l'existence d'une substance de bourrage maintenant la cuticule tendue. 
FIG. 8. - Longistriata cordicauda. $९$ : extrémité postérieure, vue latérale droite

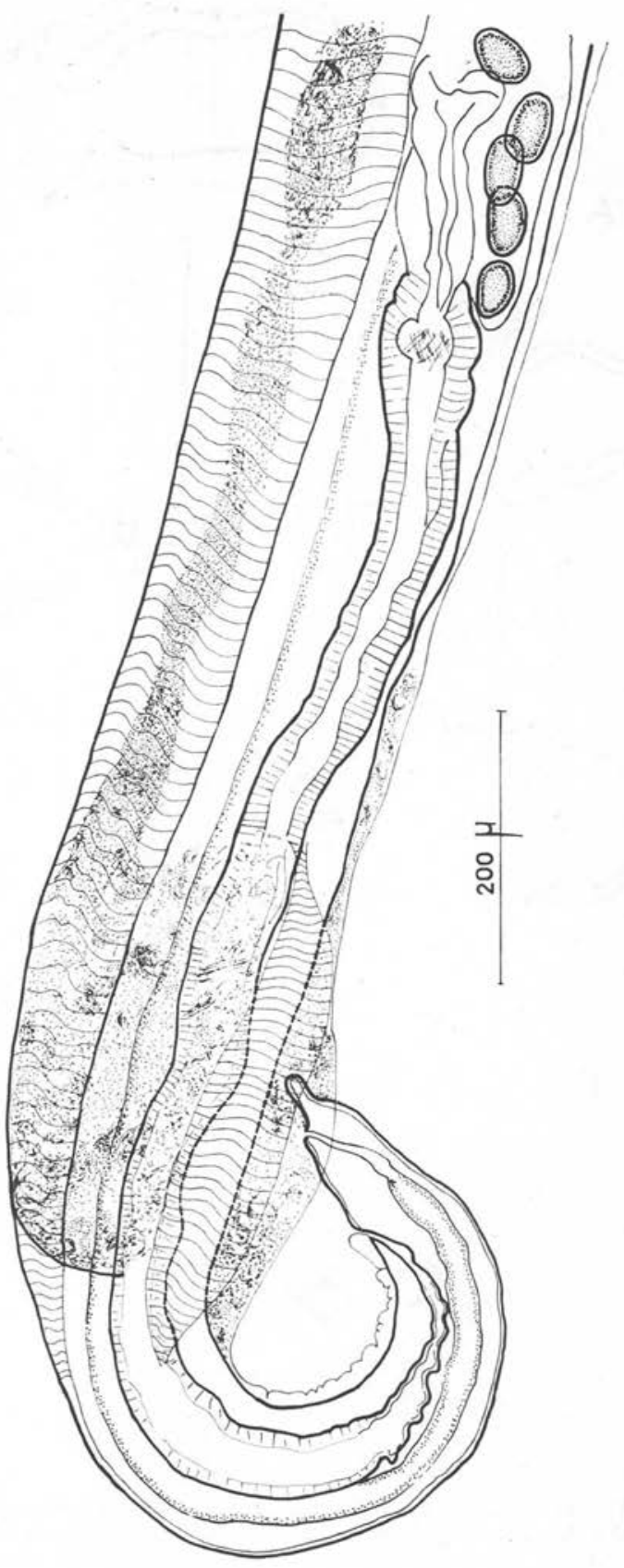




\section{B}

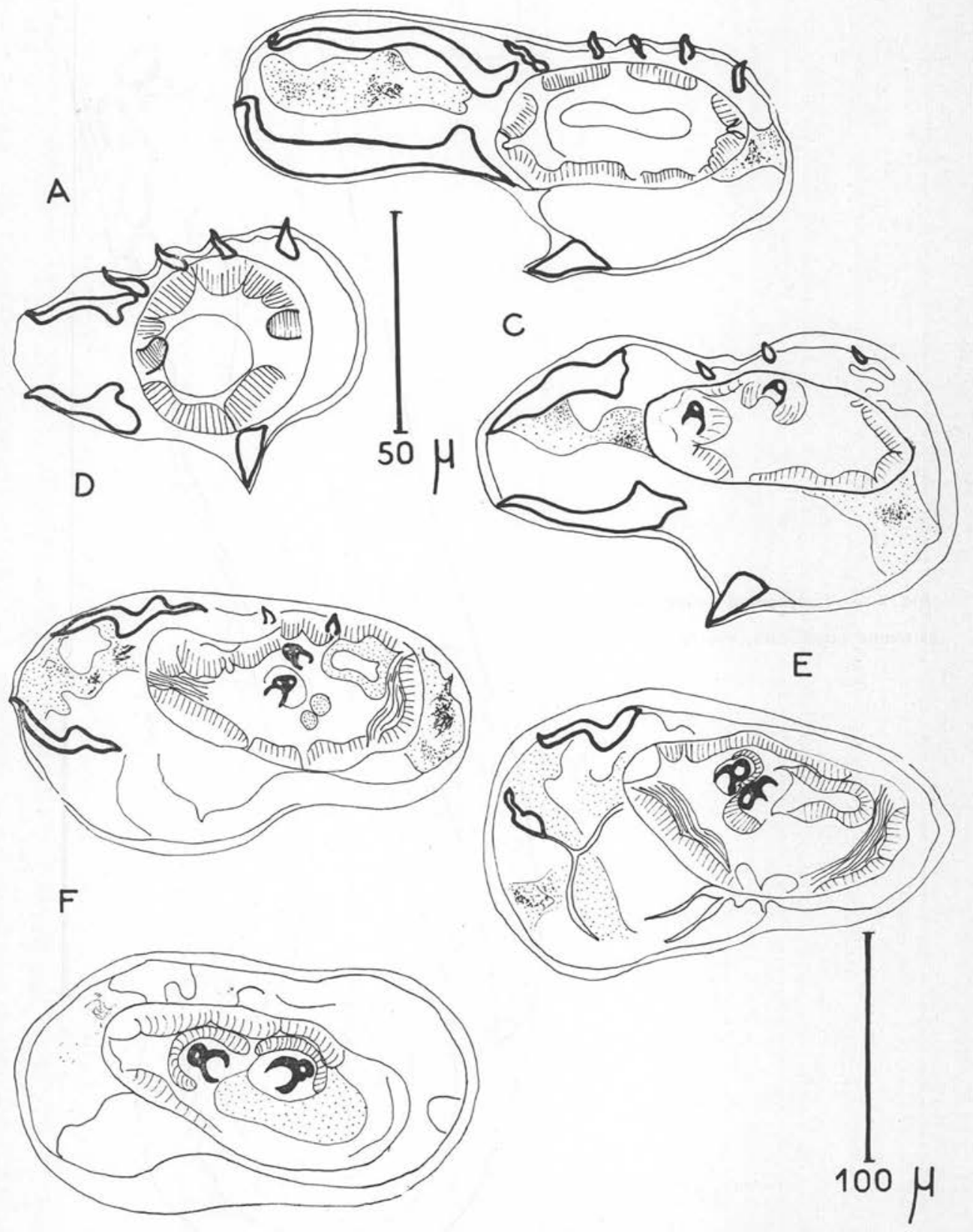

Fig. 9. - Longistriata cordicauda. $\delta$ : coupes transversales du corps. A : à $200 u$ en arrière de la tête. B : à $5.800 \mu$ en arrière de la tête. C : à $1.300 \mu$ en avant de la bourse caudale. D : à $530 \mu$ en avant de la bourse caudale. E : à $300 \mu$ en avant de la bourse caudale. F : à $120 \mu$ en avant de la bourse caudale. A: éch. $50 \mu$; B, C, D, E, F, : éch. $100 \mu$ 


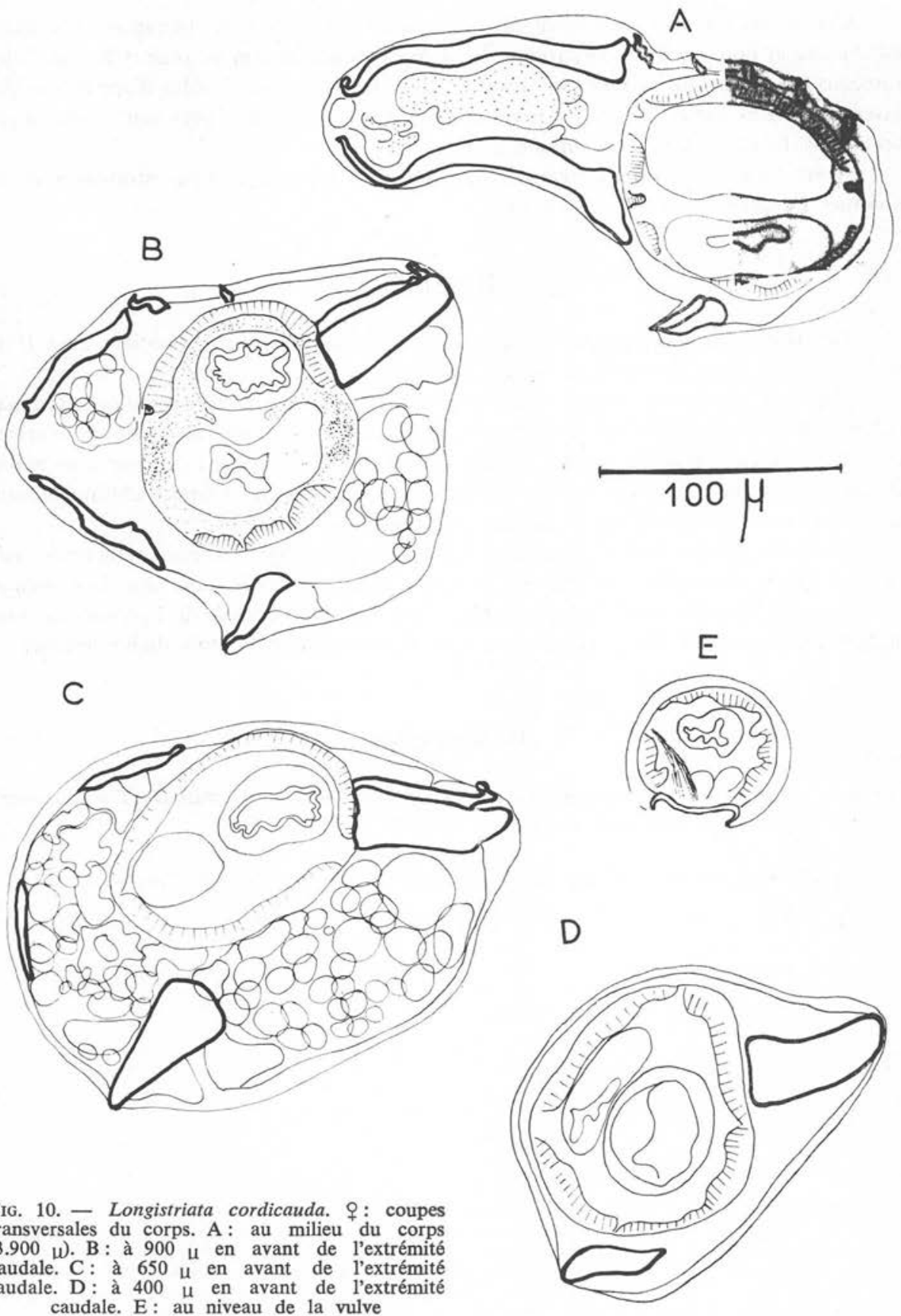


Cependant on peut facilement les différencier : chez la $\uparrow$, la dilatation cuticulaire est dorsale et non ventrale ; l'extrémité caudale est ronde et non pointue. Chez le ő, les rameaux de la dorsale ne sont pas disposés de la même façon : l'ordre d'apparition des externo-dorsales sur la dorsale est inversé. Enfin les arêtes cuticulaires sont moins nombreuses et l'arête ventrale est unique.

Nous pensons donc que notre espèce doit être séparée et nous proposons de la nommer Longistriata cordicauda $\mathrm{n}$. sp.

\section{Résumé}

Description de deux nouvelles espèces de Longistriata, vivant associées chez Hystrix cristata. L. dans la région de Saïgon au Sud Viet-Nam.

Ces deux espèces se ressemblent beaucoup. El'es sont caractérisées d'une part par l'allongement considérable des côtes dorsales, d'autre part par un système d'arêtes cuticulaires bien particulier. L'aile gauche et la cuticule dilatée à l'extrême sont maintenues rigides par l'existence d'une substance de remplissage d'aspect chitinoïde qui, sur coupe histologique, prend les mêmes colorants que les arêtes.

Ces deux espèces restent cependant faciles à séparer par plusieurs caractères : par exemple, Longistriata levanhoai n. sp. a quatre arêtes ventrales alors que Longistriata cordicauda n. sp. n'en a qu'une ; les ramifications de la côte dorsale de L. levanhoai sont dichotomiques; celles de L. cordicauda sont d'abord alternées puis dichotomiques.

\section{Bibliographie}

Chabaud (A.-G.), 1959. - Remarques sur la systématique des Nématodes Trichostrongyloidea. Bull. Soc. Zool. France, 84, 473-483.

[Laboratoire de Zoologie (Vers) Muséum National d'Histoire Naturelle], 57, rue Cuvier, F. 75 - Paris, $5^{\circ}$ 